\title{
Evaluación de la aptitud reproductiva del toro
}

\author{
Breeding Soundness Evaluation of the Bull
}

\section{Resumen}

La evaluación de la aptitud reproductiva del toro es un examen sencillo, práctico y económico que permite identificar animales subfértiles e infértiles que pueden llegar a afectar el éxito de un programa reproductivo; en consecuencia, debería realizarse de manera periódica, sin embargo, su utilización en nuestro país es baja. Esta evaluación involucra tres pasos fundamentales: el examen físico (conformación general, órganos sexuales internos y externos), la evaluación de la libido y la colecta y evaluación de semen. Se realiza una revisión de los aspectos que contemplan la evaluación reproductiva del toro, con el fin de determinar la aptitud reproductiva de los machos destinados para tal fin.

Palabras clave: Calidad seminal, Evaluación de la aptitud reproductiva, Potencial reproductivo, Toros subfértiles, Toros infértiles.

\begin{abstract}
The breeding soundness evaluation of the bull is a simple, practical and inexpensive test that allows the identification of subfertile and infertile animals that may affect the success of a breeding program. It should be performed periodically, but its use in our country is uncommon. This assessment involves three basic steps: physical examination (general shape, internal and external sex organs), evaluation of the libido, and the collection and evaluation of semen. A review of issues that includes reproductive bull evaluation in order to determine the reproductive fitness of males intended for that purpose is presented here.
\end{abstract}

Keywords: Infertile Bull, Subfertile Bull, Semen Quality, Reproductive Potential.

1 M. Sc. Universidad Nacional Abierta y a Distancia (Tunja - Boyacá, Colombia). edwin.paez@unad.edu.co.

2 Universidad Nacional Abierta y a Distancia (Tunja - Boyacá, Colombia). emma.corredor@unad.edu.co. 


\section{Introducción}

El toro, además de aportar el 50\% del material genético para la generación de un nuevo ser $(1,2)$, representa, aproximadamente, el $85 \%$ de la eficiencia del comportamiento reproductivo del hato como lo menciona Cardozo (3); en este sentido, debería ser evaluado de manera periódica, con el fin de determinar su aptitud reproductiva, sin embargo, esta práctica es poco tradicional en el país, y se aplica principalmente a aquellos reproductores destinados a los procesos de colecta con fines de criopreservación para obtención de pajillas y a toros destinados para la venta como reproductores. De acuerdo con lo señalado por Boggio (4) y por Bó y Barth (5), en procesos de servicio natural la relación toro-hembra está alrededor de 1/25 a 1/50, en tanto que en procesos de inseminación artificial esta relación puede llegar a ser de 1/10000 o superior, razón por la cual es fundamental realizar la evaluación de esos toros utilizados, ya que si falla una hembra, se puede perder una cría, pero si lo que se tiene es un toro con problema de fertilidad se puede llegar a perder hasta 40 crías o más por cada 100 hembras, tal como como plantea Boggio.

La evaluación de la aptitud reproductiva del toro es un examen que permite identificar animales subfértiles e infértiles que pueden Ilegar a afectar el éxito de un programa reproductivo en una explotación pecuaria $(6,7)$, ya que, como lo demuestran algunos estudios, aproximadamente entre el $3 \%$ y el $30 \%$ de los toros utilizados no son del todo aptos para la reproducción, pues tienen una evaluación reproductiva no satisfactoria (infértil) o poco satisfactoria (subfértil) (8).

Existen diversos métodos de evaluación; uno de los más utilizados es el señalado por Parkinson (9), citado por Chapwanya et al. (10), el cual involucra tres aspectos: examen clínico, evaluación de la libido o desempeño reproductivo y evaluación de la calidad seminal; mediante la evaluación de estos aspectos es posible determinar la aptitud y el potencial reproductivo que puede tener un toro, antes de ser incorporado a los programas reproductivos, ya sea para colecta de semen con fines de criopreservación o para los procesos de monta natural. La Sociedad Internacional de Teriogenología (SFT) ha establecido un sistema de puntuación numérica que clasifica a los toros en tres categorías: Reproductor potencialmente satisfactorio, Reproductor potencialmente insatisfactorio y Reproductor cuestionable o aplazado (11).

\section{Aspectos Generales de la Evaluación}

La evaluación de la aptitud reproductiva es un método práctico, sencillo y preciso para identificar toros que presentan subfertilidad o infertilidad y que pueden llegar a afectar la eficiencia reproductiva de un hato $(13,14,15)$. Estudios han demostrado que alrededor del $20 \%$ de los toros evaluados han tenido resultados que limitan su eficiencia reproductiva $(15,16,17)$, lo cual lleva a un bajo porcentaje de preñez en el hato. Lo anterior está acorde con lo señalado por Vejarano, Sanabria y Trujillo (14), quienes desarrollaron un estudio en el que encontraron que el $20 \%$ de los toros presentaban problemas de aplomos; el $6.6 \%$, anormalidades en órganos de los sentidos; el $18.3 \%$, anormalidades en los órganos sexuales externos, y el $16.6 \%$, anormalidades en los órganos sexuales internos, como asimetría de las ampollas y vesiculitis. Asimismo, López (18) señaló que alrededor del $20 \%$ de los reproductores evaluados en la zona centro y sur del estado de Veracruz (México) presentan resultados que los califican como insatisfactorios para el proceso reproductivo.

Esta evaluación reproductiva involucra tres pasos fundamentales: la evaluación física (conformación general, órganos sexuales internos y externos), la evaluación de la libido, y la colecta y evaluación de semen (19).

\section{Examen físico del reproductor}

El primer paso en la evaluación reproductiva del toro está representado por el examen físico general, que involucra la evaluación del estado del animal en general, y en particular de los órganos sexuales externos e internos (20). Este examen físico contempla la estimación de la condición corporal, la revisión de los ojos, los aplomos y el aparato genital externo e interno (pene, testículos, vesículas seminales), y busca descartar aquellos toros con anomalías que puedan interferir con el deseo o la capacidad de monta, Ilevando a limitar o impedir su funcionalidad reproductiva $(15,21,22)$.

El examen físico incluye la observación del toro en movimiento, de la conformación de los miembros y pezuñas y de la condición corporal general $(23,24)$. 
Condición corporal. Se debe realizar una revisión detallada del exterior del animal. Lo primero es determinar la condición corporal, que señala el estado nutricional del animal (25); la evaluación de la condición corporal en bovinos es un método utilizado para determinar el grado de reservas corporales, independientemente de la estructura, peso vivo y tamaño del animal (26). Para el caso de bovinos de tipo lechero y sus cruces se califica en escala de 1 a 5 , y para el caso de animales de tipo cárnico y sus cruces, en escala de 1 a 9 , correspondiendo 1 a un toro demasiado flaco, y 5 (tipo lechero) y 9 (tipo cárnico) a un toro obeso. Los toros no deben estar obesos, pero sí deben tener una reserva energética que les permita soportar la temporada o el proceso reproductivo (27); lo ideal es que la condición corporal del toro se encuentre en un estado intermedio, que correspondería a una escala entre 5 y 7 , para el caso del ganado de tipo cárnico como lo plantea Chenowet (28), y una escala entre 3 y 3.5 , para el caso de toros de tipo lechero; en este punto el toro puede dar una buena calidad de semen (29). Animales con sobrepeso pueden tener problemas relacionados con diminución de la libido (30).

Miembros y aplomos. Larson (31) señala que durante la época reproductiva los machos tienen que caminar mucho, por lo que la sanidad de los miembros es esencial para una monta exitosa; asimismo, para aquellos toros utilizados como donantes de semen es muy importante, ya que durante el proceso de colecta por el método de vagina artificial, todo el peso del animal estará soportado por los miembros posteriores (32), razón por la cual se requiere de unos miembros en buen estado y con el soporte suficiente para un proceso de colecta adecuado.

Para una correcta evaluación de los aplomos y de las pezuñas es necesario evaluar los toros en movimiento, debido a que es cuando se hacen más notables las cojeras, incoordinaciones y desviaciones de la postura normal $(32,33)$. De acuerdo con lo planteado por Vásquez (16), las alteraciones músculo-esqueléticas pueden limitar los movimientos y afectar el proceso de monta.

Ojos. Los ojos son otro factor importante en el proceso de evaluación, ya que el toro se guía por el estímulo visual para detectar las hembras en celo $(34,35)$; por lo tanto, son fundamentales en toros utilizados en programas de monta natural.
Se debe propugnar la selección de toros que tengan los ojos bien ubicados dentro de la órbita y que no tengan algún grado de exoftalmia, ya que es un factor predisponente de carcinoma ocular; asimismo, evitar toros que tengan cualquier tipo de patología o problema que cause disminución o pérdida parcial o total de la visión como lo señala Acuña (34).

Saco escrotal y cordón espermático. El escroto del toro debe ser observado y evaluado en su integridad; se debe realizar una palpación para determinar su suavidad al tacto y descartar la presencia de laceraciones o cicatrices que evidencien traumatismos o daños. El cordón espermático se debe palpar en toda su longitud; se debe verificar su extensión, no debe ser tan corto que pueda llevar a afectar la termorregulación, ni muy largo y colgante, que lo predisponga a constantes traumatismos. Como lo mencionan Vilanova y Ballalares (25), lo recomendable es que el fondo del escroto no sobrepase la línea de los corvejones.

Testículos y epidídimos. Los testículos, junto con el epidídimo, deben ser revisados mediante palpación detallada. Es importante descartar anomalías que pueden llegar a afectar físicamente el desarrollo de los testículos, como criptorquidia, hipoplasia, descenso incompleto y tamaño reducido de los testículos (36). La hipoplasia testicular representa el $2.7 \%$ de incidencia y es una de las principales causas de eliminación de los toros como reproductores como lo señalan Chenowet y Osborne (37). Es importante mencionar que si bien los machos con hipogonadismo pueden tener libido, raramente son fértiles por presentar oligospermia o azoospermia, con alto porcentaje de defectos morfológicos, pudiendo tener un volumen seminal normal (38). Es necesario palpar y determinar el tono, la temperatura y la simetría de los testículos para identificar posibles lesiones inflamatorias o fibróticas que puedan afectar la producción y la calidad seminal (39). Los testículos deben ser lisos y firmes al tacto y desplazarse libremente dentro del saco escrotal hacia arriba y abajo, lo cual descarta presencia de adherencias y facilita el proceso de regulación de la temperatura testicular.

Respecto a los epidídimos, se deben evaluar sus tres porciones (cabeza, cuerpo y cola), las cuales, en condiciones normales, presentan una consistencia firme y homogénea (Vilanova y Ballalares, 2005). 
Para Fordyce et al. (40) y Vilanoba y Ballanares (25) tiene gran importancia la medición de la circunferencia escrotal (CE) y la altura testicular, como elementos de gran importancia en los procesos de selección de un toro, ya que el tamaño de los testículos ha sido asociado positivamente con la producción de espermatozoides. Es importante señalar que, tal como lo manifiestan Monina et al. (41), la palpación y la medida escrotal pueden proveer información importante para investigar la capacidad reproductiva, pero no pueden detectar enfermedades potenciales; por ello se debe complementar el examen con una evaluación mediante ultrasonografía, con el fin de determinar enfermedades focales y multifocales, como abscesos y quistes.

Uno de los principales aspectos por evaluar en el proceso de revisión del escroto es la medición de la $C E$, ya que, como se ha observado en diversos estudios, las medidas de la CE están asociadas al desarrollo testicular y a las características físicas y morfológicas del semen, y pueden considerarse una característica indicativa de la edad de la pubertad en machos, presentando también correlación genética positiva con la edad de la pubertad en las hembras como lo plantean Brinks et al. (1). En este mismo sentido, se afirma que la CE y la calidad del semen están altamente correlacionadas con la fertilidad $(42,43)$, lo cual coincide con lo planteado por Gilardi, Pinho y Martins citado por Pérez et al. (44), quienes mencionan que la CE es una característica importante que ha sido utilizada durante la evaluación del potencial reproductivo en los toros adultos, ya que presenta correlaciones con determinadas características seminales. Medidas de la CE menores a $34 \mathrm{~cm}$ han sido asociadas con disminución en el porcentaje de espermatozoides normales (45).

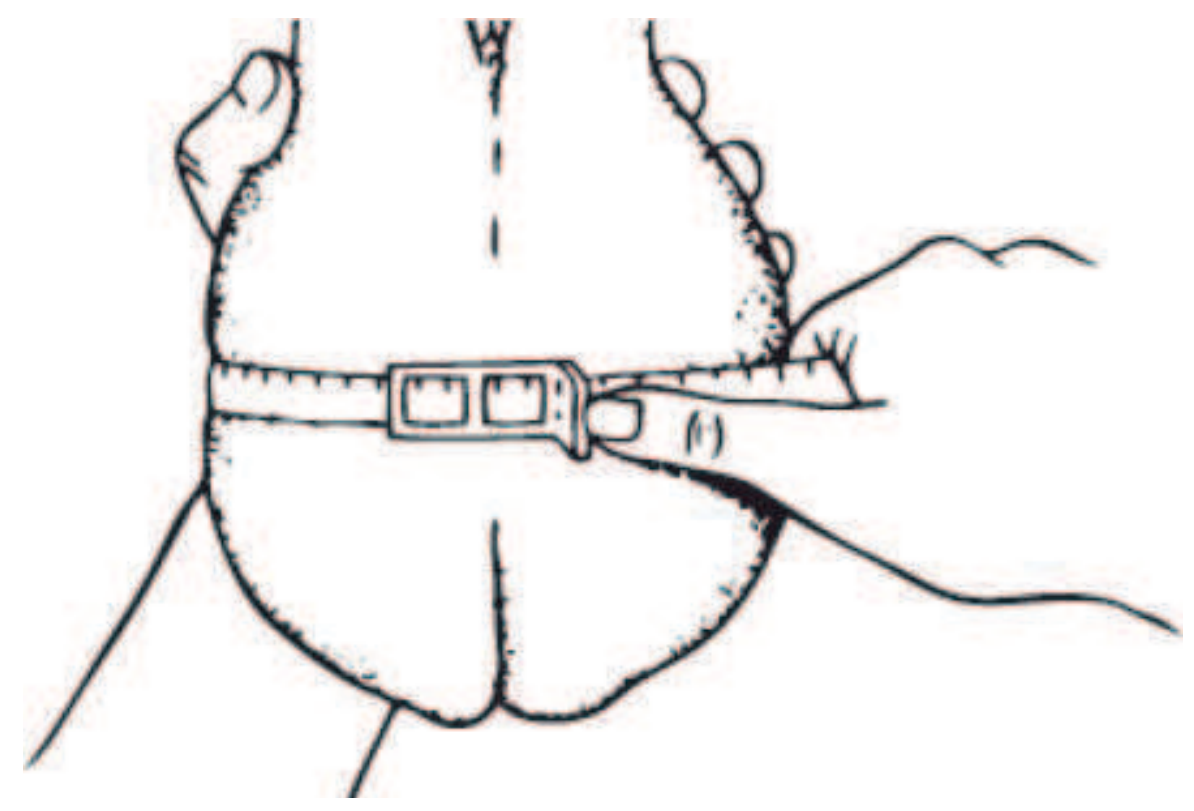

Figura 1. Medición de la circunferencia escrotal (42).

Pene y prepucio. Debe realizarse una evaluación detallada del pene y del prepucio. El pene se explora por palpación bajo la piel del abdomen, desde la inserción del escroto y en dirección al ombligo (25); debe determinarse la presencia de heridas, traumas o inflamaciones. El prepucio debe ser palpado para descartar la presencia de adherencias, heridas o hematomas; hay una característica especial en toros de tipo cebuino, y es la de tener un prepucio más penduloso, por 
lo cual son más propensos a tener lesiones que pueden llevar hasta la acrobustitis (46).

Evaluación de los órganos genitales internos. Mediante palpación rectal se deben evaluar algunos de los órganos sexuales internos; las estructuras internas palpables son: uretra pelviana, cuerpo de la próstata, vesículas seminales y ámpulas del conducto deferente; debe examinarse la consistencia de estos órganos, así como la presencia de lesiones en ellos. La lesión más común que puede encontrarse es la inflamación de la glándula, o vesiculitis, que se caracteriza por dolor a la palpación, aumento de tamaño, pérdida de las lobulaciones y adherencias (47). Si se determina la presencia de alteraciones o inflamación, es necesario descartar la existencia de un proceso infeccioso por medio de la realización de un examen de laboratorio.

Examen de laboratorio. Es importante realizar también un muestreo de sangre con el fin de descartar la presencia de enfermedades infeccionas que pueden afectar la reproducción, tales como diarrea viral bovina (DVB), brucelosis, rinotraqueítis infecciosa bovina (IBR), leptospirosis y neosporosis, entre otras (4, 48, 49). Asimismo, se recomienda realizar un raspaje prepucial para el estudio de enfermedades venéreas, como la vibriosis (Campilobacteriosis) y la tricomoniasis $(4,50)$. Estudios señalan que existen diversas enfermedades que pueden ser transmitidas a través del semen: fiebre aftosa, estomatitis vesicular, DVB, IBR, papilomatosis, leptospirosis, tuberculosis, paratuberculosis, mycoplasma, anaplasmosis, brucelosis, campilabacteriosis y tricomoniasis (51).

\section{Evaluación de la libido}

El segundo paso en un proceso de evaluación de la aptitud reproductiva del toro, esto es, de la evaluación de su libido. La libido se define como el deseo, el apetito y la disposición del macho para montar y servir a una hembra (52); implica la conducta que Ileva al macho a la monta. Este aspecto es muy importante, sobre todo en aquellos toros destinados para procesos de monta natural, donde se requiere que monte a todas las hembras aptas para tal fin, razón por la cual se exige un nivel elevado de deseo para poder llevar a cabo la monta de manera exitosa en el menor tiempo posible.
La evaluación de la libido se realiza por diversos métodos; uno de los más utilizados es el denominado método de test, consistente en asignar subjetivamente una puntuación para evaluar el grado de interés sexual y la habilidad de monta del toro (52). Según Chenowet (52) y de acuerdo con lo observado en la prueba, los toros son clasificados en cuatro grupos:

Grupo 1: Toros que sirven satisfactoriamente.

Grupo 2: Toros que hicieron intentos de monta pero no culminaron en servicio, debido a inexperiencia, falta de técnica de apareamiento o factores patológicos.

Grupo 3: Toros que montaron pero que no Ilegaron al servicio debido a falta de cooperación de la hembra. Puede reflejar factores tales como inexperiencia, baja libido o uso de una hembra inadecuada.

Grupo 4: Toros en los que no existe registro de habilidad de monta debido a falta de suficiente actividad para hacer una estimación.

Como lo señala Duchens (53) es importante evaluar de manera periódica la libido o capacidad de servicio de los toros, especialmente aquellos destinados al proceso de monta natural.

\section{Colecta y evaluación de semen}

La última fase en el proceso de evaluación de la aptitud reproductiva del toro está constituida por la colecta y la evaluación del semen. La recolección de semen en el toro puede hacerse por medio de una vagina artificial o por medio de la electroeyaculación (54). La elección del método va acorde con las características del animal del que se va a colectar, pero en general se prefiere el uso de vagina artificial para animales de tipo Bos taurus, con temperamento manso, y el uso de electroeyaculador para animales de temperamento más nervioso y agresivo, como los de tipo Bos indicus y los toros de lidia.

Lavagina artificial esel método de mayor uso, debido a su semejanza con el proceso normal de monta; sin embargo, se requiere que los toros hayan sido entrenados. El electroeyaculador está constituido por un electrodo de uso transrectal (comúnmente 
denominado bala), el cual está conectado a una batería que genera pequeños pulsos eléctricos, los cuales estimulan los órganos genitales internos, produciendo la emisión del semen, estos pulsos son dados de manera intermitente. Un aspecto que se debe tener en cuenta es que la respuesta de cada toro a la electroeyaculación es particular, razón por la cual la colecta entre uno y otro toro puede diferir en el tiempo de estimulación y en la cantidad de pulsos eléctricos necesarios para recolectar el semen (25). Este último método es más seguro para el operario, puesto que requiere la inmovilización del toro en un brete, sin embargo, puede ocurrir la contaminación del semen, ya que en algunos casos el animal no protruye el pene $y$, en consecuencia, el material seminal puede contaminarse con orina, restos y detritos celulares que puedan estar presentes en el prepucio.

Una vez se ha colectado el semen se procede a su análisis en laboratorio; allí se evalúan algunas características macroscópicas y microscópicas.

\section{Características macroscópicas}

Las características macroscópicas que se evalúan de manera general en el semen de los bovinos son: olor, color y volumen. Algunos exámenes también evalúan el aspecto y la densidad macroscópica del semen (Agüero, 2012).

Olor: es característico de la especie, no debe tener mal olor, si lo presenta puede ser indicativo de algún proceso infeccioso.

Color: se percibe mediante observación directa del eyaculado colectado (55). El color del eyaculado depende del contenido de riboflavina, y varía de acuerdo con la concentración espermática; puede ir desde un líquido blanquecino claro hasta un líquido lechoso oscuro.

Volumen: se percibe mediante observación directa del eyaculado colectado y se expresa en mililitros (55); puede estar entre $2 \mathrm{ml}$ por eyaculado en animales jóvenes y entre 4 y $12 \mathrm{ml}$ en animales adultos.

\section{Características microscópicas}

Las características microscópicas que se evalúan en el semen de bovinos son: motilidad masal, motilidad individual, morfología y viabilidad y concentración espermática (55).
Motilidad: Es uno de los parámetros más importantes en la evaluación de una muestra de semen, tal como lo señalan Hidalgo, Tamargo y Diez (56); existen diversos métodos para evaluar la motilidad, algunos computarizados, otros de tipo subjetivo, que buscan evaluar el porcentaje de espermatozoides móviles, así como el tipo de movimiento que presenta la media de una población espermática.

Se evalúan tanto la motilidad masal como la motilidad individual. A continuación se describen los métodos de mayor uso en Colombia, que si bien son subjetivos, pueden proveer una información valiosa para estimar la calidad de una muestra colectada.

La motilidad masal se puede observar colocando una pequeña gota de semen sobre una placa portaobjetos a temperatura de $37^{\circ} \mathrm{Cy}$ observándola en el microscopio en aumento de 10X o 40X; allí se observa el movimiento en masa o la denominada formación de "olas" (20). Esta motilidad masal está directamente relacionada con la concentración espermática, el movimiento progresivo y el vigor de ese movimiento; así, a mayor cantidad de espermatozoides se formará una mayor cantidad de olas; se expresa en una escala de calificación subjetiva de 1 a 5 .

La motilidad individual se determina colocando una pequeña gota de semen sobre una placa portaobjetos a temperatura de $37^{\circ} \mathrm{C}$, y sobre ella se coloca una placa cubreobjetos; se observa al microscopio, en aumento de 40X. La motilidad individual mide el porcentaje de espermatozoides que presentan un movimiento rectilíneo y continuo; el valor se expresa en porcentaje; el valor mínimo aceptado para una muestra es de $50 \%$.

Existen sistemas automáticos de medición de imágenes, los cuales se basan en la captura sucesiva de espermatozoides en movimiento; estos sistemas, denominados genéricamente CASA (Computer Assisted Motility Analysis), han automatizado y simplificado el proceso (56); su única limitante está representada por el elevado valor de los equipos, sin embargo, ya existen muchos centros que cuentan con esta tecnología, que permite una evaluación más integral y objetiva del movimiento espermático. 
Morfología y viabilidad: La evaluación morfológica de los espermatozoides es uno de los principales componentes de la evaluación de una muestra seminal. Mediante este examen se identifican las anormalidades que pueden afectar el potencial reproductivo del toro. Desde el punto de vista de la morfofisiología, las anomalías que puedan generarse se clasifican en anomalías en la cabeza, en el tracto intermedio y en la cola. Ax et al. (57) clasifican las anormalidades de los espermatozoides en tres grupos: primarias (aquellas que afectan la cabeza y el acrosoma del espermatozoide), secundarias (las que afectan la pieza o tracto intermedio) y terciarias (las que causan daño de la cola). Otra escala de clasificación, que va acorde al órgano donde puede haberse generado la anormalidad, las clasifica en anomalías primarias y secundarias (56).

La evaluación morfológica se puede realizar por medio de una tinción con eosina-nigrosina o con tinta china. En la evaluación morfológica es necesario determinar la cantidad y el tipo de anormalidades; se acepta un máximo de $30 \%$ de espermatozoides anormales.

Concentración: La concentración es un parámetro importante en la evaluación espermática, ya que existe una elevada correlación significativa entre el número de espermatozoides inseminados y la fertilidad del toro. En este punto se debe señalar que existe gran variabilidad en la concentración de un eyaculado a otro, y de un toro a otro, siendo importante conocer el número de espermatozoides por eyaculado, ya que de este parámetro depende el número de hembras por inseminar (56).

Existen diversos métodos para medir la concentración espermática; algunos de estos métodos son: la espectrofotometría, la colorimetría, la citometría de flujo y el uso de cámaras de recuento celular, como las de Bürker, Neubauer o Thoma (56). En Colombia el método más utilizado es el de la cámara de Neubauer, denominado también Hemocitómetro.

\section{Conclusiones}

Como lo señala Vásquez (16), la evaluación de la aptitud reproductiva del toro no permite predecir los índices de preñez en un hato, ya que estos dependerán de mucho factores asociados a prácticas sanitarias, nutricionales y de manejo, entre otras; sin embargo, es una herramienta que permite identificar y eliminar aquellos animales que puedan presentar problemas de infertilidad o someter a tratamiento a aquellos animales que tienen problemas de subfertilidad, pero que pueden mitigarse o eliminarse mediante tratamiento de tipo médico, convirtiéndose la evaluación reproductiva en una herramienta útil para seleccionar los mejores toros para los programas reproductivos.

Con base en los hallazgos en el examen físico del toro, la evaluación de la libido y los resultados obtenidos en la evaluación de la calidad seminal, es posible clasificar al toro en uno de tres grupos: 1) toro potencialmente satisfactorio o apto, que es aquel que tiene buena conformación física y de los órganos sexuales, buena libido y una calidad seminal buena o excelente (Tabla 1), 2) toro potencialmente insatisfactorio o no apto, que es aquel que presenta problemas a nivel fisiológico o anatómico que le impiden desarrollar el proceso reproductivo de manera satisfactoria o que tiene una baja calidad seminal y 3) toro cuestionable, dudoso o aplazado, que es aquel que presenta alguna alteración que puede ser tratada y que tras su curación será sometido a una nueva evaluación reproductiva. 
Tabla I. Valores mínimos recomendados por la Sociedad internacional de Teriogenología.

\begin{tabular}{|c|c|c|c|c|}
\hline \multicolumn{5}{|c|}{$\begin{array}{l}\text { Requerimientos mínimos para la clasificación de un toro como potencialmente satisfactorio en la } \\
\text { evaluación reproductiva (Sociedad internacional de Teriogenología) }\end{array}$} \\
\hline \multicolumn{2}{|c|}{$\begin{array}{l}\text { Circunferencia escrotal - CE } \\
\text { (mínimo recomendado) }\end{array}$} & \multicolumn{3}{|c|}{$\begin{array}{l}\text { El mínimo recomendado } \\
\text { es de } 30 \% \text { de espermatozoides con movimiento progresivo }\end{array}$} \\
\hline Edad & CE (cm) & Motilidad masal & Clasificación & Individual \\
\hline$<15$ Meses & 30 & Remolinos rápidos & Muy buena & $>70 \%$ \\
\hline$>15<18$ Meses & 31 & Remolinos lentos & Buena & $50-69 \%$ \\
\hline > $18<21$ Meses. & 32 & $\begin{array}{l}\text { Oscilación } \\
\text { generalizada }\end{array}$ & Regular & $30-49 \%$ \\
\hline$>21<24$ Meses & 33 & Oscilación esporádica & Mala & $<30 \%$ \\
\hline$>24$ Meses & 34 & & & \\
\hline $\begin{array}{l}\text { El valor mínimo rec } \\
\text { Para ser clasificado } \\
\text { los valores mínimos } \\
\text { Cualquier toro que } \\
\text { insatisfactorio o clas }\end{array}$ & $\begin{array}{l}\text { toro pote } \\
\text { os para la } \\
\text { la con los } \\
\text { dudosa o }\end{array}$ & $\begin{array}{l}\text { nente satisfactorio requi } \\
\text { ferencia escrotal, motili } \\
\text { sitos mínimos se clasific } \\
\text { ada de acuerdo con el }\end{array}$ & $\begin{array}{l}\text { un examen físi } \\
d \text { individual y } \\
\text { omo un toro p } \\
\text { erio del evalua }\end{array}$ & $\begin{array}{l}\text { atisfactorio y } \\
\text { i, morfología. } \\
\text { ialmente }\end{array}$ \\
\hline
\end{tabular}

Fuente: 22, 58, 59 .

Finalmente, es importante señalar lo mencionado por Barszcz et al. (2012), referente a los parámetros mínimos que deben tener los toros que sean utilizados en procesos reproductivos:

- $60 \%$ de espermatozoides con movimiento rectilíneo progresivo

- $80 \%$ de los espermatozoides sin anormalidades morfológicas

- 500.000 espermatozoides por mililitro

\section{Referencias}

(1) Crudeli GA, Pochon DO, Pellerano GS, García Denegri ME, Amuchastegui FL. Análisis de las variables biométricas circunferencia escrotal y volumen testicular en toros de la raza Braford. Facultad de Ciencias Veterinarias - Universidad Nacional del Nordeste. Resumen: V-051. 2005.

(2) Stahringer RC. Evaluación y manejo de toros. Instituto Nacional de Tecnología Agropecuaria. INTA. EEA Colonia Benítez. Marcos Briolini. Chaco Argentina. 2003.
(3) Sanabria Liberato RD, Trujillo Lancheros GA. Diagnóstico de la capacidad reproductiva de toros como metodología evaluativa de su eficiencia en ganaderías de tres municipios del suroccidente huilense. Facultad de Medicina Veterinaria y Zootecnia. Universidad del Tolima. Ibagué. 2002.

(4) Boggio Devincenzi JC. Evaluación de la Aptitud Reproductiva Potencial y Funcional del Toro. Capacidad de Servicio. Instituto de Reproducción Animal. Facultad de Ciencias Veterinarias. Universidad Austral de Chile. Valdivia. 2007.

(5) Bó GA, Barth A. Evaluación de la aptitud reproductiva de los toros para servicio. Rev AnG 2013; 261:32-37.

(6) Ruiz Sesma B, Ruiz Hernández $H$, Mendoza Nazar P, Oliva Llaven MA, Gutiérrez Miceli FA, Rojas Martínez RI et al. Caracterización reproductiva de toros Bos taurus y Bos indicus y sus cruzas en un sistema de monta natural y sin reposo sexual en el trópico Mexicano. Rev Cien UDO Agrí 2010; 10(1):94-102. 
(7) Godfrey RW y Dodson RE. Breeding soundness evaluations of Senepol bulls in the US Virgin Island. Theriogenology 2005; 63(3):831-840.

(8) Hernández Lagunes D. La importancia de la evaluación reproductiva del semental en el trópico. Fundación Produce Veracruz. Rev Agro 2012; 20-21.

(9) Parkinson TJ. Evaluation of fertility in natural service bulls. The Veterinary Journal 2004; 168:215-229.

(10)Chapwanya A., Callanan J., Larkin H., Keenan L. and Vaughan L. Breeding soundness evaluation of bulls by semen analysis, testicular fine needle aspiration cytology and trans-scrotal ultrasonography. Irish Veterinary Journal, 2008; 61(5): 315-317.

(11) Higdon HL, Spitzer JC, Hopkins FM, Bridges Jr WC. Outcomes of breeding soundness evaluation of 2898 yearling bulls subjected to different classification systems. Theriogenology 2000; 53 (6): 1321-1332.

(12)Sylla L, Stradaiolib G, Borgamia S, Monac M. Breeding soundness examination of Chianina, Marchigiana, and Romagnola yearling bulls in performance tests over a 10-year period. Theriogenology 2007; 67 (8): 1351-1358.

(13)Troxel R T. Breeding Soundness Evaluation for Beef and Dairy Bulls. University of Arkansas. United States. Department of Agriculture, and County Governments Cooperating. Sf.

(14)Vejarano OA, Sanabria RD, Trujillo GA. Diagnóstico de la capacidad reproductiva de toros en ganaderías de tres municipios del alto Magdalena. Rev. MVZ Córdoba 2005; 10 (2):648-662.

(15)García Paloma JA. Valoración de la aptitud reproductiva de toros de monta natural. Área de Genética y Reproducción. Centro de Biotecnología Animal. SERIDA-Gijón. Tecnología Agroalimentaria. Boletín Informativo del SERIDA 2013; 11:33-38.

(16)Vásquez LA. Evaluación del Potencial Reproductivo del Toro. Director Instituto de Reproducción Animal e Inseminación Artificial. Maracay, Edo. Aragua. Venezuela. 2009.
(17)Orantes Zebadúa MA, Manzur Cruz A, Ruiz Rojas JL, Sánchez Muñoz B, Cruz López JL, Ortega Jiménez E et al. Evaluación de sementales bovinos en el programa "Ganado Mejor" de la región Centro de Chiapas, México. Queh Cien Chiapas 2010;1 (10):34-38.

(18)López Mata, S.A. Análisis retrospectivo de la calidad seminal de sementales bovinos en la zona centro y sur del estado de Veracruz. Universidad Veracruzana. Facultad de Medicina Veterinaria y Zootecnia. México. 2007.

(19)LeaMaster BR, DuPont MW. Bull Power: Examination of Beef Cattle Bulls for Breeding Soundness. College of Tropical Agriculture and Human Resources (CTAHR). Department of Human Nutrition, Food and Animal Sciences. Livestock Management. L-17. April 2007.

(20)Angelino Olivera JN. Manual de evaluación de semen en bovinos. Universidad Veracruzana. Facultad de Medicina Veterinaria y Zootecnia. 2009.

(21)Duchens M, De los Reyes M. Examen de fertilidad potencial a toros. Universidad de Chile. Facultad de Ciencias Veterinarias y Pecuarias. Departamento de Fomento de la producción animal. Unidad de reproducción. 2012.

(22)Whittier WD, Bailey T. Predicting Bull Fertility. Extension Veterinarians, Cattle, Department of Large Animal Clinical Sciences, VirginiaMaryland Regional College of Veterinary Medicine, Virginia Tech. May 1, 2009.

(23)Engelken T.J. The development of beef breeding Bulls. Theriogenology 2008; 70 (3):573-575.

(24)Duchens M. Examen de fertilidad para selección en toros de carne. Rev. TecnoVet 1999; 5(2).

(25)Vilanoba LT, Ballarales PP. La evaluación andrológica: justificación y métodos. Manual de Ganadería Doble Propósito. Universidad Centroccidental "Lisandro Alvarado", Decanato de Ciencias Veterinarias, Barquisimeto, Estado Lara, Venezuela. 2005.

(26)Salgado R, Vergara O, Simanca J. Relaciones entre peso, condición corporal y producción de leche en vacas del sistema doble propósito. Rev. MVZ Córdoba 2008; 13 (2):1360-1364. 
(27)Loyola Oriyés CJ, Fiss Poll AA, Vázquez Montes de Oca R, Ramírez Oriyés JA. Influencia de la condición corporal en la libido sexual de receladores vacunos con fimosis artificial. Rev. prod. Anim 2014; 26 (3).

(28)Stahringer RC. Condición Corporal en el Manejo del Rodeo de Cría. Instituto Nacional de Tecnología Agropecuaria. INTA. EEA Colonia Benítez. Marcos Briolini. Chaco Argentina. 2003.

(29)Guarié EA. Nutrición del toro y calidad seminal. Universidad de Buenos Aires. Facultad de Ciencias Veterinarias. 2013.

(30) Lozano H. Factores que afectan la calidad seminal en toros. Rev. Med. Vet. Zoot. 2009; 56: 258-272.

(31)Larson B. Toros: examen sanitario y de aptitud reproductiva. Kansas State University. American Angus Journal. 2007.

(32)Morillo M, Salazar S, Castillo E. Evaluación del potencial reproductivo del macho bovino. INIA. Centro Nacional de Investigaciones Agropecuarias. Maracay. Venezuela. 2012.

(33) Bavera GA, Peñafort C. Examen reproductivo completo en toros. Curso de Producción Bovina de Carne, cap. VI. Vet-uy. 2003.

(34)Capandeguy Istebot JI, Mattos Amorim B. Principales hallazgos en la evaluación andrológica en toros de campo. Universidad de la República. Facultad de Veterinaria. Uruguay. 2014.

(35)Dávalos CW, Ortiz TJJ. Prueba de libido en toros Nelore. (Cabaña El Trébol y estancia Los Ángeles, provincia Cordillera de Santa Cruz). Facultad de Ciencias Veterinarias, Uagrm. Bolivia. 2005.

(36)Silva C, Delgado R, Magaña J, Reyes A. Anomalías del desarrollo testicular y escrotal en toros de tres razas en el sureste de México. Av. Inv. Agro 2008; 12 (3).

(37)Rodríguez Márquez JM, Madrid Bury N, Urdaneta de Romero A, Aranguren JA, Quintero A. Análisis morfométrico del epidídimo en toros jóvenes mestizos 5/8 Holstein y 5/8 pardo suizo con testículos pequeños. Revista científica FCVLUZ 2000; 10 (6) 458-467.
(38)Facultad de Ciencias Veterinarias-FCV. UBA. Cátedra de Semiología - Medicina I. Semiología del aparato reproductor macho. 2013.

(39)Caspe G. Medidas Clínico sanitarias para la cría bovina. Instituto Nacional de Tecnología Agropecuaria-INTA. Octubre de 2010.

(40)Fordyce G, McGowan MR, Lisle A, Muller T, Allen J, Duff $C$ et al. Scrotal circumference of Australian beef bulls. Theriogenology 2013; 81(6):805-812.

(41)Monina MI, Heritier JM, Della Croce MR, Galetti EJR, lerace A.J.M, Juan FM et al. Evaluación ultrasonográfica de las gonadas del toro. Universidad Nacional de la Pampa. Facultad de Ciencias Veterinarias. Argentina. 2000.

(42)Perry G, Patterson D. Determining reproductive fertility in Herd bulls. University of MissouriColumbia. Departament of Animal Sciences. 2011.

(43)Guzmán CR. Evaluación seminal en toros por métodos manuales o computarizados. Universidad de la República. Facultad de veterinaria. Uruguay. 2013.

(44) Pérez Osorio J, Chacón Jaramillo L, Otero Arroyo RJ, Cardona Álvarez J, Andrade Souza F. Relación entre la circunferencia escrotal, el crecimiento testicular y parámetros de calidad de semen en toros de raza Guzerat, desde la pubertad hasta los 36 meses de edad. Rev Med Vet. 2014; 27:73-87.

(45)Waldner CL, Kennedy RI, Palme CW. A description of the findings from bull breeding soundness evaluations and their association with pregnancy outcomes in a study of western Canadian beef herds. Theriogenology 2010; 74(5):871-883.

(46)Escamilla AR. Aplicación de clorhidrato de xilacina $(0.05 \mathrm{mg} / \mathrm{kg})$ en toros como facilitador de la colecta de semen con el método de electroeyaculador. Universidad de San Carlos de Guatemala. Facultad de Medicina Veterinaria y Zootecnia. Escuela de Medicina Veterinaria. Guatemala, noviembre 2005.

(47)Madrid Bury N. Evaluación de la aptitud reproductiva del toro. En: C. González-Stagnaro 
(Ed), Reproducción Bovina. Fundación Girarz, Maracaibo-Venezuela. Cap. XII: 264-279. 2001.

(48)Sprott LR, Field RW. Reproductive diseases in cattle. Texas Agriculture Extension Service. Texas A\&M University System. 2012.

(49)Walker B. Diseases causing reproductive losses in breeding cattle. Veterinary Officer, Gunnedah. Rev. first edn 2005.

(50)Wikse S. Management of infectious reproductive diseases in beef cattle herds. Beef Cattle Clinician and Extension Veterinarian. Texas A\&M University. College Station. 2005.

(51)Peña Joya MA, Góngora A, Jiménez C. Infectious agents affecting fertility of bulls, and transmission risk through semen. Retrospective analysis of their sanitary status in Colombia. Rev Colomb Cienc Pecu 2011; 24:634-646.

(52)Chenowet, PJ. Impulso sexual del toro y comportamiento reproductivo. Large Animal Clinical Sciences, College of Veterinary Medicine, Kansas State University, Manhattan, Kansas, USA. Traducido por C. Jiménez Escobar, Fac. de Medicina Veterinaria y de Zootecnia, Univ. Nacional de Colombia, Bogotá. 2003.

(53)Vera Castillo CA. Evaluación de la validez de la cría y análisis de semen para predecir la fertilidad del toro. Universidad de Cuenca. Facultad de Ciencias Agropecuarias. Escuela de Medicina Veterinaria y Zootecnia. 2011.
(54)Jiménez Escobar C. Técnicas de congelación y sexado del semen bovino y su importancia en reproducción bovina. Universidad Nacional de Colombia. Facultad de Medicina Veterinaria y Zootecnia. 2010.

(55)Agüero G. Evaluación de las Características Seminales de Sementales Bovinos mediante el Analizador Seminal Computarizado (CASA). Universidad Central de Venezuela. Facultad de Ciencias Veterinarias. Instituto de Reproducción Animal. Enero, 2012.

(56) Hidalgo Ordoñez CO, Tamargo Miguel C, Diez Monforte C. Análisis del semen bovino. Tecn Agro. 2005; 2:39-43.

(57)Purwantara B, Arifiantini RI, Riyadhi M. Sperm morphological assessments of friesian holstein bull semen collected from three artificial insemination centers in Indonesia. J. Indonesian Trop.Anim.Agric. 2010; 35 (2).

(58)Alexander JH. Bull breeding soundness evaluation: A practitioner's perspective. Theriogenology 2008; 70 (3): 469-472.

(59)Kennedy SP, Spitzer JC, Hopkins FM, Higdon HL, Bridges Jr WC. Breeding soundness evaluations of 3648 yearling beef bulls using the 1993 . Society for Theriogenology Guidelines 2002; 58 (5):947-961.

(60)Barszcz K, Wiesetek D, Wasowicz M, Kupczyaska M. Bull Semen Collection and Analysis for Artificial Insemination. Journal of Agricultural Science 2012; 4(3):1-10. 